\title{
Produção da ficotoxina diarreica ácido ocadaico associada à microalga Dinophysis acuminata (Ehremberg 1839) na baía de Sepetiba, RJ e sua implicação para a saúde pública
}

\section{Production of diarrhoetic phycotoxin okadaic acid associated with the microalgae Dinophysis acuminata (Ehremberg 1834) in Sepetiba bay, RJ, and your implication in public health}

\author{
Vanessa de Magalhães Ferreira, ${ }^{*, *}$ Gesilene Mendonça de Oliveira, ${ }^{*}$ Milena Marcela Domingues Pereira, ${ }^{*}$ \\ Pedro Paulo de Oliveira da Silva, ${ }^{*}$ Hélcio Rezende Borba, ${ }^{* * *}$ Aderbson Jorge Lourenço, ${ }^{*}$ Patrícia Ferreira da Silva, ${ }^{*}$
}

\begin{abstract}
Resumo
Microalgas constituem o principal alimento para moluscos bivalvos. Porém no fitoplâncton podem ser encontradas microalgas nocivas como Dinophysis, implicadas na produção da toxina ácido okadaico (AO). A baía de Sepetiba, RJ, apresenta áreas adequadas à malacocultura, principalmente na região da ilha Guaíba. Nessa região localiza-se um dos maiores bancos naturais do mexilhão Perna perna neste estado, que fornece sementes para cultivos nas baías de Sepetiba e llha Grande. O AO, principal causador do Envenenamento Diarréico por Moluscos, apresenta como efeito agudo sintomatologia gastrintestinal. Como efeito crônico relata-se a promoção de tumores (estômago e intestinos). Este estudo objetivou detectar o AO e identificar Dinophysis spp. na região estudada. Coletaram-se mexilhões em bancos naturais (ilhas Guaíba e Madeira), para a detecção do AO e amostras de fitoplâncton para a identificação das microalgas durante a primavera/verão 2003/2004. A análise por Cromatografia Líquida de Alta Eficiência detectou o AO, em baixas concentrações, em todas as amostras. Identificaram-se cinco espécies de Dinophysis, das quais $D$. acuminata apresentou a maior abundância relativa para o gênero no período analisado. Os resultados sugerem um perfil toxígeno de baixo potencial, porém constante. Nessa circunstância, consumidores regulares de moluscos poderiam estar expostos ao perigo representado pelos efeitos crônicos desta ficotoxina.
\end{abstract}

Palavras-chave: cromatografia líquida de alta eficiência, microalgas nocivas, envenenamento diarréico por moluscos.

\begin{abstract}
The major source of food for bivalve molluscs is microalgae. In the phytoplancton can be found potentially toxic microalgae as Dinophysis spp., often involved in the production of the toxin okadaic acid (OA). Sepetiba Bay, RJ, presents appropriated sites to malacoculture, mainly in the region of the Guaiba Island. In this region is located one of the largest natural bed of mussel Perna perna in the state, going to seed crops in the Sepetiba and Ilha Grande bays. OA, which is the most implicated toxin of Diarrhoetic Shellfish Poisoning, presents gastrointestinal symptoms as acute effect. Chronic effects related are promotions of tumors in stomach and intestines. The present study aimed to detect OA and identify Dinophysis present in studied sites. Mussels were collected from natural beds (Guaiba and Madeira islands) for the detection of OA and phytoplankton samples collects were carried out for the identification of harmful microalgae, during spring and summer 2003/2004. Chemical analysis using High Performance Liquid Chromatography detected the presence of OA in all samples in low concentrations. Five species of potentially toxic Dinophysis were identified, from which $D$. acuminata showed higher relative abundance to genera in the period. The results suggest a constant low profile of the toxigenic potential. Thus regular consumers of shellfish could be exposed to the chronic effects of this phycotoxin.
\end{abstract}

Keywords: HPLC, hamful microalgae, diarrhoetic shellfish poisoning.

\footnotetext{
*Universidade Federal Rural do Rio de Janeiro. Laboratório de Toxinas Marinhas (ToxMar);

${ }^{* *}$ Faculdade de Oceanografia, UERJ. Av. São Francisco Xavier, 524, 4 andar, sala 4023, Maracanã, CEP 20550-013

***Instituto de Biologia, UFRRJ Rodovia BR 465 Km7 Campus Universitário Seropédica RJ CEP: 23890-000;

Universidade Federal Rural do Rio de Janeiro. Laboratório de Toxinas Marinhas (Departamento de Tecnologia de Alimentos/ Instituto de Tecnologia/ UFRRJ Rodovia BR 465 Km7 Campus Universitário Seropédica RJ CEP: 23890-000 E-mail: toxmar.ufrrj@gmail.com
} 


\section{Introdução}

A síndrome Envenenamento Diarréico por Moluscos (EDM) constitui um dos maiores problemas de saúde pública, em nível mundial, associado ao consumo de moluscos bivalvos. Dinophysis acuminata é o principal agente etiológico implicado e possui ampla distribuição latitudinal. Essa espécie de dinoflagelado causa grandes prejuízos econômicos também à malacocultura com a suspensão da comercialização em locais como Espanha e Nova Zelândia (FAO, 2004), mas também recentemente no Brasil (Proença et al., 2007).

Geralmente Dinophysis spp. ocorrem em baixas abundâncias, cerca de 1-10\% da comunidade fitoplanctônica mas, raramente, podem formar florações típicas conforme o ocorrido em Santa Catarina em janeiro de 2007. São ditas microalgas potencialmente nocivas, pois sua capacidade toxígena pode ser expressa ou não em um determinado momento. Microalgas deste gênero podem produzir toxinas mesmo em baixas densidades, poucas centenas de células por litro de água do mar (Reguera, 2002).

Ficotoxinas são substâncias biologicamente ativas e o ácido okadaico é a principal toxina diarreica associada ao EDM. Os sintomas (dores de cabeça e abdominais, vômito, diarreia) surgem poucas horas após o consumo de moluscos contaminados com a ficotoxina, apresentando LOAEL $1 \mu \mathrm{g}$ AO.kg de peso corpóreo-1 (Toyofuku, 2006). Duram aproximada-mente três dias, com ou sem tratamento médico. Recomenda-se tratamento de suporte para reposição de líquidos e eletrólitos (FAO, 2004). Porém, há fortes evidências de que o consumo constante de moluscos contaminados (com concentrações inferiores às necessárias ao desenvolvimento do EDM) exponha o consumidor aos efeitos crônicos desta ficotoxina, carcinogênese de estômago e intestinos (Cordier et al., 2000).

Na baía de Sepetiba encontram-se grandes bancos naturais do mexilhão Perna perna, importante reservatório de sementes para a malacocultura do litoral sul fluminense. A ficotoxina $A O$ já foi detectada em mexilhões capturados na baía de Sepetiba (Oliveira et al., 2005), em ostras de Recife (Souza et al., 2007) e em cultivo na Ilha Grande (Lourenço et al., 2007) e Santa Catarina (Proença et al., 2007). O presente trabalho objetivou detectar a ficotoxina $\mathrm{AO}$ em mexilhões de bancos naturais e identificar as microalgas do gênero Dinophysis presentes na área estudada.

A Comissão Oceanográfica Intergovernamental (UNESCO) recomenda que ambientes onde haja extração ou cultivo de moluscos sem monitoramento higiênico-sanitário sejam considerados áreas de risco para a saúde pública. Urge, portanto, o imediato estabelecimento do Programa Nacional de Controle Sanitário de Moluscos Bivalvos para o pleno e seguro desenvolvimento da malacocultura no litoral sul fluminense.

\section{Material e métodos}

\section{Área de estudos}

A baía de Sepetiba é um ambiente estuarino, parcialmente misturado, que funciona conjugado à baía da Ilha Grande (Signorini, 1980). Apresenta áreas com características fisiográficas e oceanográficas adequadas ao desenvolvimento da malacocultura (enseadas abrigadas, rasas, águas com temperaturas amenas, boa circulação) principalmente próximo à saída da baía em contato como oceano Atlântico (Figura 1).

Figura 1: Baía de Sepetiba, adaptada de IBGE (escala 1:100 000), fonte EMBRAPA Monitoramento por Satélite. P01: ilha da Madeira e P02: ilha Guaíba. I, II e III áreas hidrobiológicas: fundo da baía, área do canal de maré e área de influência oceânica, respectivamente.

É um ambiente mesotrófico, que oferece abundante alimento aos moluscos (Ferreira, 2004). Os pontos selecionados para a coleta de mexilhões e fitoplâncton localizavam-se em áreas hidrobiologicamente distintas (Sema, 1998): na ilha da Madeira (área I, parte mais interna da baía e mais impactada) e Guaíba (área III, menos impactada, maior influência do oceano Atlântico) como visto na Figura 1. Foram realizadas três coletas dentro do período de primavera/verão 2003/2004.

\section{Fitoplâncton: coleta e identificação}

As microalgas foram coletadas durante a preamar com rede de plâncton, malha de $20 \mu \mathrm{m}$ (ALFAKIT®) para a obtenção de amostras concentradas (qualiquantitativas) uma vez que Dinophysis apresenta baixa abundância. Foi realizado arrasto vertical, integrando toda a coluna d'água, pois dinoflagelados podem se agregar em determinadas profundidades em condições de estabilidade da coluna d'água (Reguera, 2002). As amostras foram fixadas em formaldeído para a identificação em microscópio invertido de microscopia óptica de campo claro TELAVAL 3 (Carl Zweiss, Germany), baseada 
em análise morfométrica usando material bibliográfico de Steidinger e Tangen (1997) e Balech (2002). A contagem para o cálculo da abundância relativa foi realizada em toda a câmara de sedimentação segundo metodologia descrita em Uehlinger (1964) apud Vianna (2003).

\section{Ácido ocadaico}

Cada coleta consistia em três amostras (50 animais cada) em cada um dos pontos estudados, num total de nove amostras. Os animais seguiam refrigerados para o laboratório, onde eram removidas as sujidades. As glândulas digestivas eram então seccionadas e congeladas para posterior extração da toxina.

A extração da toxina foi realizada segundo SIGMA (1993) e a derivação foi otimizada por Silva et al. (2003). Alíquotas de $20 \mu \mathrm{l}$ da solução derivada foram injetadas no sistema de Cromatografia Líquida de Alta Eficiência (Waters Associats, Inc, Miliford, M.A. - USA) equipado com detector de fluorescência Waters (modelo 420) com excitação 333nm e emissão

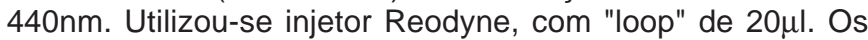
dados obtidos foram analisados pelo integrador registrador Waters Data Module (modelo 740). As condições cromatográficas utilizadas foram: fase móvel com acetonitrila e água ultrapura (85:15 v/v); fluxo de $1 \mathrm{ml}$.minuto-1; coluna cromatográfica Microsorb C18 (4,6 X 15 mm) Microsorb-VMTM $10 \mu \mathrm{m}$ (Varian-USA), a temperatura ambiente.

\section{Resultados e discussão}

Análise qualiquantitativa do microfitoplâncton revelou a presença de cinco espécies de Dinophysis: $D$. acuminata, $D$. caudata, $D$. fortii, $D$. tripos e $D$. rotundata, todas relatadas como potencialmente toxígenas (ZINGONE, 2004). Na ilha Guaíba foram encontradas as maiores abundâncias e todas as espécies. Na ilha da Madeira apenas $D$. acuminata e $D$. caudata foram identificadas. Dinophysis é um gênero típico de águas neríticas e oceânicas, sendo que $D$. acuminata pode ser encontrada em águas costeiras eutróficas (Steidinger e Tangen, 1997).

Nas coletas de primavera a dominância da comunidade foi exercida por diatomáceas, com 54,70\% na ilha Guaíba e $55,90 \%$ na ilha da Madeira, as variáveis abióticas mensuradas (dados não apresentados nesse artigo) demonstraram uma situação muito homogênea, bem misturada. No entanto, segundo Ferreira (2004), no verão foi observada uma heterogeneidade nas características oceanográficas (águas tipicamente costeiras na ilha da Madeira e neríticas na ilha Guaíba). Na ilha da Madeira houve dominância de dinoflagelados $(94,35 \%)$ enquanto na ilha Guaíba as diatomáceas permanecerem dominantes $(57,72 \%)$. Os dados encontram-se de acordo com o verificado por Vianna (2003) para a mesma região.

Dinophysis acuminata (Figura 2) foi a espécie mais abundante do gênero, na ilha Guaíba no verão (1,51\%). Na primavera as abundâncias foram semelhantes $0,2 \%$ e $0,4 \%$ para Guaíba e Madeira, respectivamente. $D$ tripos (0,06\%) foi encontrada só na primavera e $D$. fortii $(0,76 \%)$ só no verão, ambas apenas na Guaíba. D. rotundata ocorreu em ambas estações, mas foi mais abundante no verão $(0,61 \%)$.

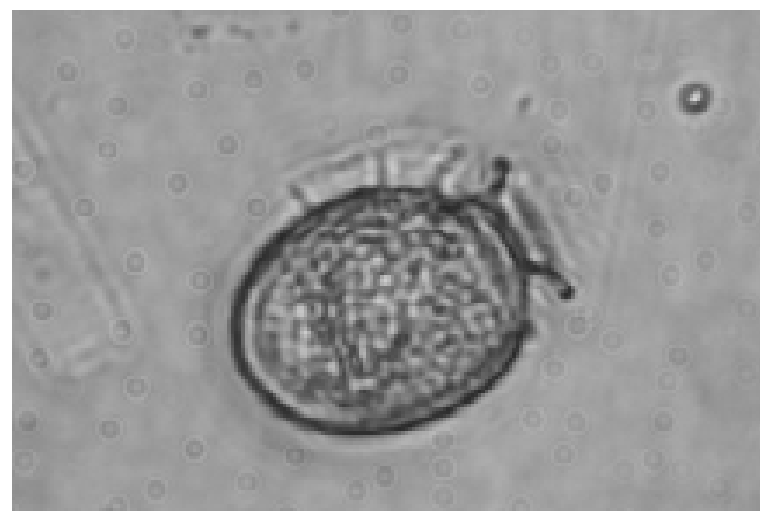

Figura 2: Dinophysis acuminata, microscopia de campo claro, aumento 400x. Exemplar de verão, ilha Guaíba. Fotomicrografia FERREIRA, V. M.

A ficotoxina diarreica ácido ocadaico foi encontrada em todas as amostras analisadas. Porém o nível de contaminação foi baixo, na ordem de poucos nanogramas de AO por grama de glândula digestiva de molusco. Durante o verão foram encontradas as maiores concentrações detectadas neste estudo: 23,07 ng AO para ilha da Madeira e $24 \mathrm{ng}$ AO para ilha Guaíba (Figura 3, item B).

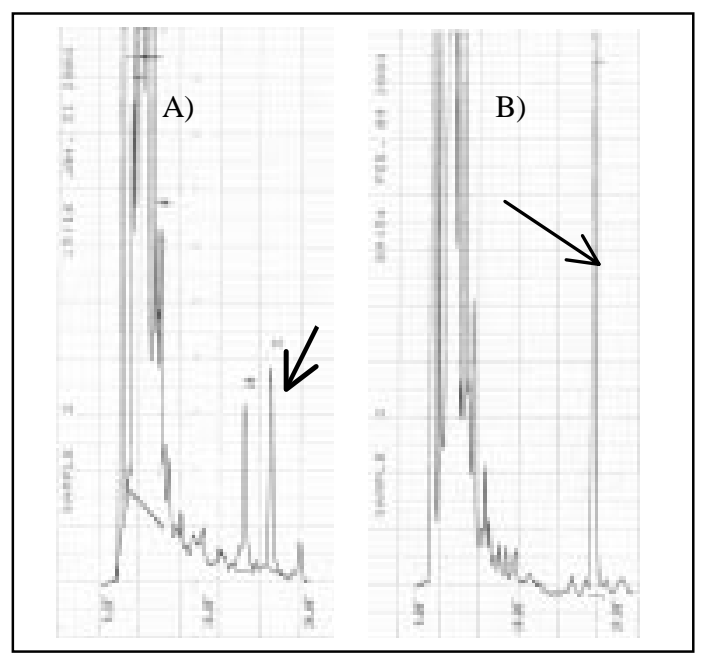

Figura 3: Cromatogramas das amostras de mexilhões da ilha Guaíba: A) amostra de primavera, perfil G03c, contendo ácido ocadaico com tempo de retenção de 16,581 minutos e área 48580, presença de 3,45 ngAO.g1 glândula digestiva e B) amostra de verão, perfil G04b, contendo ácido ocadaico com tempo de retenção de 18,188 minutos e área 115865 , presença de 14,62 ngAO.g-1 glândula digestiva.

Na primavera, a concentração média da toxina ácido ocadaico foi 14,83ng AO.g-1 de glândula digestiva na ilha da Madeira e 6,37ng.g-1 glândula digestiva na ilha Guaíba (Figura 3, item A). O nível máximo permitido, em países onde há legislação que regulamente os valores permitidos de ficotoxinas em moluscos, é de $2 \mu \mathrm{g} \mathrm{AO.g-1} \mathrm{de} \mathrm{glândula} \mathrm{digestiva} \mathrm{(Toyofuku,}$ 2006). Partindo dessa premissa, os mexilhões coletados nas ilhas Guaíba e Madeira, em relação a ficotoxina ácido ocadaico, estariam apropriados para o consumo durante todo o período analisado. Oliveira et al. (2005) encontraram a 
mesma ordem de grandeza das concentrações de AO para mexilhões capturados na baía de Sepetiba em 2000. Tais resultados sugerem que o perfil toxígeno de Dinophysis spp. seja baixo e constante na baía de Sepetiba.

\section{Conclusões}

1 As concentrações de ácido ocadaico, nas amostras analisadas, encontram-se abaixo do limite necessário ao desenvolvimento dos sintomas clássicos de EDM e foram associados à Dinophysis acuminata.

2 As baixas concentrações de ácido ocadaico, encontradas pelo presente trabalho, alertam para o risco de carcinoma

\section{Agradecimento}

À CAPES, pela cessão da bolsa.

\section{Referências}

BALECH, E. Dinoflagelados tecados tóxicos del Cono Sur Americano. In: Floraciones algales nocivas en el Cono Sur Americano. SAR, E. A.; FERRARIO, M. E.; REGUERA, B. (Ed.) Madrid: Instituto Español de Oceanografía, 2002, p. 125-144.

CORDIER, S.; MONFORT, M.; MIOSSEC, L.;RICHARDSON, S.;BELIN, C. Ecological analysis of digestive cancer mortality related to contamination by diarrhetic shellfish poisoning toxins along the coasts of France. Environmental Research Section A, v. 84, p. 145-150, 2000.

FOOD AND AGRICULTURE ORGANIZATION-FAO. Marine Biotoxins. FAO Food and Nutrition Paper, n. 80. Roma: FAO, 2004. 294 p.

FERREIRA, V. M. Detecção de ácido ocadáico produzido por Dinophysis spp. (Ehremberg, 1839), em mexilhões Perna perna (Linné, 1758), em situação de primavera e verão, nas ilhas Guaíba e Madeira, baía de Sepetiba, Rio de Janeiro. 2004. 53 f. Dissertação (Mestrado em Microbiologia Veterinária). Universidade Federal Rural do Rio de Janeiro, Seropédica, 2004.

LOURENÇO, A. J.; FERREIRA, V. M.; SILVA, P. P. O.; ROSA, C. A. R.; DIREITO, G. M.; OLIVEIRA, G. M. Evidência de depuração natural da toxina diarreica ácido ocadaico em mexilhões Perna perna (Linnè, 1758) cultivados em fazenda de maricultura na baía de llha Grande, Angra dos Reis, RJ. Revista Brasileira de Ciência Veterinária, v. 14, n. 2, p. 91-94, 2007. Disponível em: <http://www.uff.br/rbcv/site/ index.php/numeros/view/6>. Acesso em: 02 jul. 2008.

OLIVEIRA, G.M.; SILVA, P.P.O.; ROSA, C.A.R.; BASTOS, R.A.; FERREIRA, V.M. RODRIGUES. E.S. Detección de ácido okadaico por cromatografía líquida de alta eficiencia en mariscos (Perna perna) capturados en la bahía de Sepetiba. Revista Alimentaria, v. 366, p. 56-61, 2005.

PROENÇA, L.A.O.; SCHRAMM, M.A.; TAMANAHA, M.S.; ALVES, T.P. Diarrhoetic shellfish poisoning (DSP) outbreak in Subtropical Southwest Atlantic. Harmful Algae News, v. 33, p. 19-20, 2007

REGUERA, B. Establecimiento de un programa de seguimiento de microalgas tóxicas. In: SAR, E. A.; FERRARIO, M. E.; REGUERA, B. (Ed.). Floraciones algales nocivas en el Cone Sur Americano. Vigo: Instituto Español de Oceanografia, 2002, p. 21-54. no trato gastrintestinal de consumidores regulares de molusco, devido ao alto potencial carcinogênico do AO.

3 A identificação da presença de espécies potencialmente produtoras de outras toxinas diarreicas - dinophysistoxina (DTX-1), D. fortii, D. tripos e $D$. rotundata e pectenotoxina (PTX-2) D. fortii - apontam para a possibilidade destas toxinas também estarem presentes nas águas da baía de Sepetiba. Recomenda-se a realização de pesquisa para averiguar se há produção destas toxinas.

4 Urge a implantação do Programa Nacional de Controle Higiênico Sanitário de Moluscos Bivalvos para que sejam assegurados tanto a sanidade dos animais quanto a segurança do alimento produzido.
SECRETARIADE ESTADODEMEIOAMBIENTEDOESTADODORIODE JANEIRO - SEMA. Uma Avaliação da Qualidade das Águas Costeiras do Estado do Rio de Janeiro. Rio de Janeiro: Fundação Estudos do Mar, 1998. 194 p.

SIGMA - SIGMA CELL CULTURE COMPANY. Okadaic Acid Detection Kit. Canada: USA, 1993. p. 7.

SIGNORINI, L.C. A Study of the Circulation in Bay of Ilha Grande and of Sepetiba. Part I. A Survey of the Circulation based on Experimental Field Data. Boletim do Instituto Oceanográfico de São Paulo, p. 1521,1980 .

SILVA, P. P. O. ; SANTANA, D. M. N.; OLIVEIRA, G. M.; ROSA, C. A. R. Otimização de metodologia para detecção de ácido okadaico por cromatografia líquida de alta eficiência em moluscos bivalvos. Revista Higiene Alimentar, v. 17, n. 114/115, p. 29-33, 2003.

SOUZA, J. C. R; BARROS, G. C.; SILVA, P. P. O.; FERREIRA, V. M.; OLIVEIRA, G. M. Primeira detecção de ácido okadaico em ostras (Crassostrea rhizophorae) coletadas no canal de Santa Cruz, Itapissuma, Pernambuco. Revista Higiene Alimentar, v. 21, n. 149, p. 17-21, 2007.

STEIDINGER, K. A.; TANGEN, K. Dinoflagellates. In: TOMAS, C. R. (Ed.). Identifying Marine Phytoplancton. San Diego: Academic Press, 1997. p. 397-583.

TOYOFUKU, $\mathrm{H}$. Joint FAO/WHO/IOC activities to provide scientific advice on marine biotoxins (research report). Marine Pollution Bulletin, v. 52, p. 1735-1745, 2006.

VIANNA, S. C. O Microfitoplâncton da Baía de Sepetiba: composição e densidade relativa na primavera de 2001 e outono de 2002. 2003. 28 f. Trabalho de Conclusão de Curso (Graduação). Curso de Bacharelado em Biologia Marinha, Universidade Federal do Rio de Janeiro, Rio de Janeiro. 2003.

ZINGONE, A. Dinoflagellates-Ordem Dinophysiales. In: MOESTRUP, $\varnothing$. (Ed.). IOC Taxonomic Reference List of Toxic Algae. Intergovernmental Oceanographic Commission of UNESCO. Disponível em: <http://www.ioc.unesco.org/hab/data.htm>. Acesso em: 18 out. 2004. 